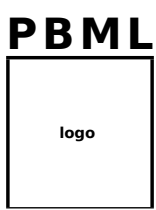

The Prague Bulletin of Mathematical Linguistics

NUMBER ??? AUGUST 2011 1-10

\title{
eppex: Epochal Phrase Table Extraction for Statistical Machine Translation
}

\author{
Česlav Przywara, Ondřej Bojar \\ Charles University in Prague \\ Faculty of Mathematics and Physics \\ Institute of Formal and Applied Linguistics
}

\begin{abstract}
We present a tool that extracts phrase pairs from a word-aligned parallel corpus and filters them on the fly based on a user-defined frequency threshold. The bulk of phrase pairs to be scored is much reduced, making the whole phrase table construction process faster with no significant harm to the ultimate phrase table quality as measured by BLEU. Technically, our tool is an alternative to the extract component of the phrase-extract toolkit bundled with Moses SMT software and covers some of the functionality of sigfilter.
\end{abstract}

\section{Motivation}

Phrase tables in Statistical Machine Translation (SMT) systems generally take the form of a list of pairs of phrases $s$ and $t, s$ being the phrase from the source language and $t$ being the phrase from the target language, along with scores that should reflect the goodness of translating $s$ as $t$. The standard approach to obtain such scores is to use maximum likelihood probability of the phrase $t$ given the phrase $s$ and vice versa. The probabilities $p(s \mid t)$ and $p(t \mid s)$ are often referred to as forward and reverse translation probabilities.

To estimate $p(s \mid t)$ and $p(t \mid s)$, frequency counts $C(t, s), C(s)$ and $C(t)$ are usually collected from the entire training corpus. For substantial coverage of source and target languages, such corpora are often very big so all phrase pairs and their counts cannot fit in the physical memory of the computer. To overcome this limitation, phrase table construction methods often simply dump observed phrases to local disk and sort and 
count them on disk. This approach allows to construct phrase tables of size limited only by the capacity of the disk. The obvious drawback of this solution is that much more time is needed to build the table.

Moses (Koehn et al., 2007), an open-source SMT toolkit with a full set of tools required for SMT system training, adheres to this concept. Both of the two main components used to construct phrase tables (extract to observe phrases and scorer to score them) treat their input as an unbounded stream of data, keeping only limited span of this stream in physical memory and using local disk for temporary storage.

It is known that phrase table quality is not strictly determined by its size. Johnson et al. (2007) presented a method for the reduction of phrase table size causing no harm to translation quality as measured by BLEU (Papineni et al., 2002). Their method employs significance testing of phrase pair co-occurrence in the parallel corpus to distinguish between valuable phrase pairs and random noise. Because significance testing of phrase pair co-occurrences is based on their frequencies, the method was designed as a post-processing filter applied to a finished phrase table. The phrase table extraction process and its runtime requirements are unaffected by this method.

In this paper, we present eppex, a tool designed as a drop-in alternative for extract component in Moses training. Like extract, our tool extracts phrase pairs from a wordaligned parallel corpus. Unlike extract, eppex filters out phrase pairs with frequency below a user-defined threshold. As a result, the subsequent sorting and scoring have to process a reduced set of phrase pairs, so the whole phrase table extraction pipeline requires less time to finish.

In the rest of the paper we present the implementation details and results of the experiments aiming at comparison of the standard approach, the standard approach with additional significance filtering and the epochal extraction with respect to translation quality and runtime performance.

\section{Implementation}

Our tool, just like the extract component, processes the input parallel corpus in a single pass. Our implementation reuses the code from extract that implements the extraction of individual phrase pairs from word-aligned parallel corpora as proposed by Och and Ney (2003). In contrast to extract, extracted phrase pairs are not immediately printed to a temporary storage on the disk, but instead they are fed into an algorithm that on the fly filters out low frequency items.

To carry out the filtration within manageable memory demands, we employ an algorithm for approximate frequency counting proposed by Manku and Motwani (2002). Their Lossy Counting algorithm expects two user-defined thresholds: support $s \in(0,1)$ and error $\epsilon \in(0,1)$, such that $\epsilon \ll s$. At any point of time (after being fed with $\mathrm{N}$ items) the algorithm can output the list of items with their approximate frequencies and guarantee the following:

- All items whose true frequency exceeds $s \mathrm{~N}$ are output (no false negatives). 
- No item whose true frequency is less than $(s-\epsilon) \mathrm{N}$ is output (few false positives).

- Estimated frequencies are less than the true frequencies by at most $\in \mathrm{N}$.

- The space used by the algorithm is $\mathrm{O}\left(\frac{1}{\epsilon} \log \epsilon \mathrm{N}\right)$.

\subsection{Lossy Counting Algorithm}

The Lossy Counting algorithm conceptually divides the incoming stream of items into epochs of fixed size $w=\left\lceil\frac{1}{\epsilon}\right\rceil$ (thus the name epochal extraction). In order to deliver the frequency estimates, the algorithm maintains a data structure D consisting of triples $(e, f, \Delta)$, where $e$ is an element from the stream, $f$ is its estimated frequency and $\Delta$ is the maximum possible error in $f$. When a new item $e$ arrives, a lookup for $e$ in $\mathrm{D}$ is performed. If $e$ is already present, its frequency $f$ is incremented by one. Otherwise a new triple $(e, 1, T-1)$ is added to $D$, where T denotes the ID of current epoch (with IDs starting from 1 ).

At the end of each epoch (determined by $N \equiv 0 \bmod w$ ), the algorithm prunes off all items whose maximum true frequency is small. Formally, at the end of the epoch $\mathrm{T}$, all triples satisfying the condition $f+\Delta \leqslant T$ are removed from $D$. When all elements in the stream have been processed, the algorithm returns all triples $(e, f, \Delta)$ where $f \geq(s-\epsilon) N$.

The idea behind the algorithm is that frequent elements show up more than once within each epoch so their frequencies are increased enough to survive the filtering.

\subsection{Memory Management}

To optimize the usage and access speed of the memory, we implement a couple of tricks. First, we explicitly store the vocabulary of the phrases read so far. The phrases are then represented as vectors of word indices instead of full strings. (The vocabulary is not subject to pruning at epoch boundaries for efficiency reasons.)

Second, we take advantage of the fact the vocabulary size of MT corpora usually is on the order of millions. We therefore represent words as 4-byte integers allowing to store up to 4 billions of word types. Using directly the pointer to the string representation of the word would be more expensive in a 64-bit environment.

Third, we optimize the process of memory allocation for newly created word types by using memory pools as implemented in the Boost library.

\subsection{Usage}

Eppex is implemented as $\mathrm{C}++$ program and does not require any special libraries to compile and run, except for moderately recent version of $g c c$. The authors did not attempt to compile eppex on Windows, but the code is free of system-dependent hacks, so porting to Windows should be fairly straightforward.

Eppex input and output format is fully compatible with that of extract. We also keep the command line syntax very similar. The main change is that instead of the max- 
phrase-length parameter, one has to specify the $\epsilon$ and s values for the Lossy Counting algorithm. A different pair of thresholds can be given for each phrase pair length ${ }^{1}$ allowing for a more fine-grained pruning.

The command line syntax is:

eppex tgt src align extract lossy-counter [lossy-counter-2 [...]]।

[orientation [ --model [wbe|phrase|hier]-[msd|mslr|mono] ]]

Every lossy-counter specifies the error and support for the Lossy Counting algorithm for phrases of a length within an interval. The parameter takes the form length:error:support, where length is either a number or an interval specification and error and support are two floats. For example, to keep in all phrase pairs with length 1 and prune all phrase pairs of length from 2 to 4 with $\epsilon=2 \times 10^{-7}$ and $s=8 \times 10^{-7}$, two lossy counters must be declared as: 1:0:0 2-4:2e-7:8e-7. ${ }^{2}$

Phrases of length not covered by any lossy-counter are not extracted at all, effectively setting also the max-phrase-length.

No defaults for lossy-counters are provided, because reasonable settings heavily depend on the corpus. Eppex at its end and also a faster single-purpose tool counter report the total number of extracted phrases of all lengths, allowing to set the thresholds.

\section{Experiments}

We compared our tool against two other methods of phrase table construction that were already introduced in the beginning of the paper:

1. the extract component of Moses toolkit, i.e. the baseline,

2. the sigfilter program, which is a re-implementation of significance testing phrase table filter described by Johnson et al. (2007) and is also bundled with Moses.

The baseline scenario has no options to adjust: all phrase pairs extracted from the corpus are included in the final phrase table. When applying sigfilter, at least one of two options has to be set: the cutoff threshold or the pruning threshold. By setting the cutoff threshold to $n$, all but the top $n$ phrase pairs, sorted by the forward probability $P(t \mid s)$, will be removed. Johnson et al. (2007) recommend the cutoff of 30 . The pruning threshold determines the minimum level of negative-log-p-value that a particular phrase pair $(s, t)$ has to reach under Fisher's exact test that calculates probability of observed two by two contingency table based on frequency counts $C(s), C(t)$ and $C(s, t)$. A particularly interesting settings for this threshold are values $\alpha-\epsilon$ and $\alpha+\epsilon$, where $\alpha=\log (\mathrm{N})$ and $\epsilon$ is appropriately small positive number. The former is the largest

\footnotetext{
${ }^{1}$ Phrase pair length is defined as the length of the longer of the phrases.

${ }^{2}$ All phrases of length $2-4$ are stored together in one counter. To treat them separately, the counter has to be split in three: $2: 2 e-7: 8 e-7 \quad 3: 2 e-7: 8 e-7 \quad 4: 2 e-7: 8 e-7$.
} 
threshold that results in keeping all the 1-1-1 phrase pairs ${ }^{3}$ in the table, whereas the latter is the smallest threshold that results in all such phrase pairs being removed.

With eppex, a separate lossy counting may be instantiated for each phrase pair length, allowing to filter them with different values of $s \mathrm{~N}$ (positive) and $(s-\epsilon) \mathrm{N}$ (negative) thresholds. Depending on the corpus, the values of support and error have to be adjusted. The effect of sigfilter with $\alpha \pm \epsilon$ pruning may be approximated: setting support s such that $s \mathrm{~N}<1$ will preserve all of the 1-1-1 phrases (like in $\alpha-\epsilon$ ), while setting support $s$ and error $\epsilon$ to satisfy $(s-\epsilon) \mathrm{N}>1$ will result in their complete removal (like in $\alpha+\epsilon)$.

\subsection{Data}

We evaluate the extraction methods on English-Czech translation trained on the corpus CzEng (Bojar and Žabokrtský, 2009) with a few additions and a large Czech language model. See Mareček et al. (2011) for the exact setup of the system "cu-bojar".

The complete parallel corpus for our experiments with phrase extraction is $8.4 \mathrm{M}$ sentence pairs; 107.2M English and 93.2M Czech tokens.

Our tuning and testing data come from the WMT 2011 Translation Task ${ }^{4}$.

\subsection{Benchmarking}

The training process of Moses SMT takes place in nine steps. ${ }^{5}$ The steps cover the whole training pipeline including word alignment, lexical table construction, phrase table construction and more. The phrase table construction itself is done in two steps, phrase extraction and phrase scoring, which might be even further split into following substeps: (1) phrase extraction that produces direct and reverse phrase table halves (without scores yet); (2) gzipping, (3) sorting and (4) scoring of the direct table; (5) gzipping, (6) sorting and (7) scoring of the reverse table; (8) sorting of the scored reverse table; (9) consolidation of the scored direct and reverse tables; (10) gzipping of the consolidated phrase table.

The default implementation in Moses training script train-model.perl does not use any parallelization of the sequence (steps $2-4$ and $5-7$ could be run in parallel). The gzipping in steps 2 and 5 is somewhat dubious but everybody seems to use it. ${ }^{6}$

We benchmark phrase table construction by measuring CPU time, wall clock time and memory requirements of all the substeps. To measure the memory with a reasonable precision, we save a copy of stat and status files from / $\mathrm{proc} /[\mathrm{pid}] /$ directory of the measured process(es) every second.

\footnotetext{
${ }^{3}$ A phrase pair $(s, t)$ is called $1-1-1$, if $C(s)=1, C(t)=1$ and $C(s, t)=1$.

${ }^{4}$ http://www. statmt.org/wmt11/translation-task.html

${ }^{5} \mathrm{http}: / /$ www. statmt . org/moses/?n=FactoredTraining . HomePage

${ }^{6}$ We discovered that the option -dont-zip of train-moses.perl has been broken since it was introduced.
} 
We run all the steps on a single machine for comparability of the results. Although the machine is a standard node in a cluster, we keep jobs of other users away by reserving all memory of the machine for our job. The machine runs the 64-bit version of Ubuntu 10.04 server edition on 2 Core 4 AMD Opteron $2.8 \mathrm{Ghz}$ processors with $32 \mathrm{~GB}$ of RAM in total. All the input and output files were read and written to a locally mounted hard disk.

\section{Results}

Table 1 presents all the experiments and their settings. We compare the baseline, the recommended default settings for significance filtering and two eppex runs: one with mild pruning that left in all shorter phrase pairs (denoted as eppex 1-in) and one harsher that filters out all phrase pairs with single occurrence only (eppex 1-out).

\begin{tabular}{|c|c|}
\hline Name & Description \\
\hline baseline & standard Moses pipeline with extract component \\
\hline eppex 1 -in & $\begin{array}{l}\text { the pipeline with eppex: all phrase pairs of length } 1-3 \text { kept in, } \\
\text { longer phrase pairs pruned with max. positive threshold of } 8\end{array}$ \\
\hline eppex 1-out & $\begin{array}{l}\text { the pipeline with eppex: all single-occurring phrase pairs re- } \\
\text { moved, phrase pairs pruned with max. positive threshold of } 8\end{array}$ \\
\hline sigfilter a-e & baseline followed by sigfilter with pruning threshold $\alpha-\epsilon$ \\
\hline sigfilter $a+e$ & baseline followed by sigfilter with pruning threshold $\alpha+\epsilon$ \\
\hline sigfilter 30 & baseline followed by sigfilter with cutoff threshold of 30 \\
\hline
\end{tabular}

Table 1. List of the experiments and their settings

\subsection{Translation quality}

We evaluate translation quality automatically using BLEU. The complete SMT system includes also a language model (always identical) and a distortion model. For eppex setups, the distortion model was always built from the same (pruned) set of phrase pairs as the phrase table. In each setup separately, model weights are optimized using Moses MERT.

Table 2 presents BLEU scores obtained in the experiments and the respective phrase table sizes. For both of the test sets, the top three results were obtained in baseline, sigfilter 30 and eppex 1-in experiments, but all the differences are rather small and could be also attributed to the randomness of MERT. ${ }^{7}$ The baseline scenario ranked best on wmt11 set (BLEU score 18.22), while the eppex 1-in scenario topped on wmt10 set

\footnotetext{
${ }^{7}$ We did not invest the computing resources necessary to estimate the confidence bounds covering optimizer instability (Clark et al., 2011).
} 


\begin{tabular}{|c|c|c|c|c|}
\hline & \multicolumn{2}{|c|}{ Final phrase table size } & \multicolumn{2}{c|}{ BLEU score } \\
\hline Experiment & phrase pairs & .gz file size & wmt10 & wmt11 \\
\hline baseline & $153.6 \mathrm{M}$ & $3.68 \mathrm{~GB}$ & 17.36 & $\mathbf{1 8 . 2 2}$ \\
sigfilter 30 & $137.0 \mathrm{M}$ & $3.36 \mathrm{~GB}$ & 17.48 & 18.13 \\
sigfilter a-e & $92.4 \mathrm{M}$ & $2.39 \mathrm{~GB}$ & 17.23 & 17.87 \\
eppex 1-in & $57.1 \mathrm{M}$ & $1.28 \mathrm{~GB}$ & $\mathbf{1 7 . 6 0}$ & 18.10 \\
sigfilter a+e & $35.0 \mathrm{M}$ & $0.86 \mathrm{~GB}$ & 17.31 & 17.99 \\
eppex 1-out & $14.4 \mathrm{M}$ & $0.33 \mathrm{~GB}$ & 17.23 & 17.94 \\
\hline
\end{tabular}

Table 2. Phrase table sizes and BLEU scores for all experiments

(BLEU score 17.60). However, the phrase table extracted in eppex 1-in occupied only 1.28 GB space on disk, being less than half of the size of the baseline (3.68 GB) and sigfilter 30 (3.36 GB) phrase tables.

Harsher pruning in both eppex and sigfilter can reduce the phrase table size up to one tenth of the baseline with only negligible loss in BLEU.

\subsection{Memory and time requirements}

Table 3 presents in detail physical memory peaks for all experiments. In eppex scenarios, it is the epochal extraction itself that is the most demanding part of the pipeline, consuming 19.2 GB (1-in) and 16.7 GB (1-out) of memory. In all the other experiments the memory consumption is considerably lower and the scorer component is responsible for the peak, except for the cases when $\alpha \pm \epsilon$ significance filtering is applied.

\begin{tabular}{|c|r|c|}
\hline Experiment & VM peak & in step \\
\hline baseline & $1.1 \mathrm{~GB}$ & scoring-e2f \\
sigfilter 30 & $1.1 \mathrm{~GB}$ & scoring-e2f \\
sigfilter a-e & $5.4 \mathrm{~GB}$ & sigfilter \\
eppex 1-in & $19.2 \mathrm{~GB}$ & phr-ext \\
sigfilter a+e & $5.4 \mathrm{~GB}$ & sigfilter \\
eppex 1-out & $16.7 \mathrm{~GB}$ & phr-ext \\
\hline
\end{tabular}

Table 3. Virtual memory peaks for all experiments

Table 4 compares CPU usage and wallclock times of all the substeps of the pipeline in baseline and eppex scenarios. While the initial extraction of phrase pairs takes much longer with eppex than with extract, subsequent steps finish much quicker in the eppex scenario: total time required is less than half in case of 1-in pruning and less than 1/4 


\begin{tabular}{|c|cc|cc|cc|}
\hline & \multicolumn{2}{|c|}{ baseline } & \multicolumn{2}{c|}{ eppex 1-in } & \multicolumn{2}{c|}{ eppex 1-out } \\
\hline step & CPU & wallclock & CPU & wallclock & CPU & wallclock \\
\hline phr-ext & 1145 & $\mathbf{1 1 5 2}$ & 4346 & $\mathbf{4 3 6 0}$ & 4349 & $\mathbf{4 3 6 1}$ \\
gzip-f2e & 590 & 662 & 183 & 226 & 86 & 114 \\
gzip-e2f & 593 & 641 & 185 & 276 & 87 & 132 \\
sort-f2e & 653 & 2257 & 304 & 822 & 196 & 564 \\
sort-e2f & 628 & 2844 & 315 & 810 & 199 & 567 \\
score-f2e & 10516 & 10795 & 4493 & 4531 & 371 & 372 \\
score-e2f & 9400 & 9622 & 2867 & 2902 & 340 & 340 \\
sort-inv & 358 & 1569 & 129 & 129 & 21 & 22 \\
cons & 754 & 1361 & 269 & 269 & 65 & 66 \\
pt-gzip & 791 & 881 & 258 & 259 & 65 & 65 \\
\hline TOTAL & 25428 & 31784 & 13349 & 14584 & 5779 & 6603 \\
hh:mm:ss & $7: 03: 48$ & $\mathbf{8 : 4 9 : 4 4}$ & $3: 42: 29$ & $\mathbf{4 : 0 3 : 0 4}$ & $1: 36: 19$ & $\mathbf{1 : 5 0 : 0 3}$ \\
\hline
\end{tabular}

Table 4. CPU and wallclock times (in seconds) of the phrase table construction.

\begin{tabular}{|c|cc|cc|cc|}
\hline Sigfilter settings & \multicolumn{2}{|c|}{-1 a+e } & \multicolumn{2}{c|}{-1 a-e } & \multicolumn{2}{c|}{-n 30 } \\
\hline & CPU & wallclock & CPU & wallclock & CPU & wallclock \\
\hline sigfilter alone & 18635 & 18248 & 19252 & 18449 & 2105 & 1141 \\
\hline total incl. baseline & 44063 & 50032 & 44680 & 50233 & 27533 & 31784 \\
hh:mm:ss & $12: 14: 23$ & $13: 53: 52$ & $12: 24: 40$ & $13: 57: 13$ & $7: 38: 53$ & $9: 08: 45$ \\
\hline
\end{tabular}

Table 5. CPU and wallclock times (in seconds) of significance filtering. The total includes the time of baseline extraction: 7:03:48 (CPU) and 8:49:44 (wallclock).

in case of 1-out pruning compared to the baseline. Partial parallelization of the baseline extraction as suggested above decreases the gains but eppex still remains safely faster, esp. if eppex used the same optimization.

Significance filtering requires an additional amount of time (see Table 5) on top of the baseline extraction. The most striking difference is between sigfilter $\alpha+\epsilon$ and eppex 1-out. They are comparable in terms of BLEU score and phrase table size but sigfilter took almost 14 hours while eppex 1-out finished in less than 2 hours.

\section{Related and Future Work}

We point out that the idea of using approximate frequency counting algorithms in the field of NLP is not new. Goyal et al. (2009) used approximate n-gram frequency counts to build language models, which they then applied successfully in SMT achieving no significant loss in BLEU. 
A recent feature of Moses is incremental training (probably related to the experiments by Levenberg et al. (2010)) aiming at the reduction of time required to incorporate recent data into already trained models. The entire source and target corpora are indexed in suffix arrays along with their alignments. Phrase extraction and scoring happens on the fly when phrases are needed in translation, completely eliminating the expensive batch retraining. Because the reduction of training time is the main advantage of eppex, we intend to perform a comprehensive comparison of this feature with batch retraining utilizing eppex.

The epochal extraction in eppex also lends itself to incremental extraction: only the counts in the current epoch have to be stored and reloaded when the model is to be extended by new data. The setting of the thresholds, however, would require a fairly large amount of training data to be processed in the first batch to estimate the values that will lead to sufficient saturation of phrase table. Our initial experiment suggests that for short phrase pair lengths it is beneficient to use no pruning at all.

Hardmeier (2010) presented memscore, an open-source tool to score phrases in memory that acts as a faster drop-in replacement for the sorting and the scorer in the pipeline. By combining memscore and eppex into a single phrase extraction tool a further speed up of phrase table construction process might be achieved.

Furthermore, we expect the benefits of eppex to be even more significant when confronted with larger training corpora, therefore we are in the process of its evaluation on the $10^{9}$ French-English corpus available as part of WMT training data.

\section{Conclusions}

We presented eppex, a tool for extraction of phrase pairs from word-aligned parallel corpus capable of phrase pairs filtering on the fly based on a user-defined threshold. Eppex is a drop-in alternative of extract component in Moses training toolkit. Our tool is ready to use and it is available in Moses SVN trunk (in scripts/training/eppex).

We compared our tool against the baseline extraction and another common approach to phrase table filtration. By using eppex for phrase extraction we were able to obtain translation quality comparable to the baseline, while at the same time both the (wallclock) training time and phrase table size have been reduced by more than a half or up to one tenth with harsher pruning. Although memory requirements are significantly increased, they still lie within manageable limits.

\section{Acknowledgment}

This work has been supported by the grants EuroMatrixPlus (FP7-ICT-2007-3-231720 of the EU and 7E09003 of the Czech Republic), P406/10/P259, and MSM 0021620838. 


\section{Bibliography}

Bojar, Ondřej and Zdeněk Žabokrtský. CzEng0.9: Large Parallel Treebank with Rich Annotation. Prague Bulletin of Mathematical Linguistics, 92:63-83, 2009. ISSN 0032-6585.

Clark, Jonathan H., Chris Dyer, Alon Lavie, and Noah A. Smith. Better hypothesis testing for statistical machine translation: Controlling for optimizer instability. In Proc. of ACL/HLT, pages 176-181, Portland, Oregon, USA, June 2011. URL http: //www. aclweb. org/anthology/P11-2031.

Goyal, Amit, Hal Daumé, III, and Suresh Venkatasubramanian. Streaming for large scale NLP: language modeling. In Proc. of HTL/NAACL, pages 512-520, Boulder, Colorado, 2009. URL http: //portal. acm. org/citation. cfm?id=1620754 . 1620829.

Hardmeier, Christian. Fast and Extensible Phrase Scoring for Statistical Machine Translation. The Prague Bulletin of Mathematical Linguistics, 93:79-88, 2010.

Johnson, J Howard, Joel Martin, George Foster, and Roland Kuhn. Improving Translation Quality by Discarding Most of the Phrasetable. In Proc. of EMNLP and Computational Natural Language Learning, pages 967-975, 2007.

Koehn, Philipp, Hieu Hoang, Alexandra Birch, Chris Callison-Burch, Marcello Federico, Nicola Bertoldi, Brooke Cowan, Wade Shen, Christine Moran, Richard Zens, Chris Dyer, Ondrej Bojar, Alexandra Constantin, and Evan Herbst. Moses: Open Source Toolkit for Statistical Machine Translation. In Proc. of ACL (Demonstration Session), pages 177-180, 2007.

Levenberg, Abby, Chris Callison-Burch, and Miles Osborne. Stream-based translation models for statistical machine translation. In Proc. of HTL/NAACL, pages 394-402, Los Angeles, California, 2010. URL http://portal . acm. org/citation. cfm?id=1857999. 1858061.

Manku, Gurmeet Singh and Rajeev Motwani. Approximate Frequency Counts over Data Streams. In Proceedings of the 28th International Conference on Very Large Data Bases, 2002.

Mareček, David, Rudolf Rosa, Petra Galuščáková, and Ondřej Bojar. Two-step translation with grammatical post-processing. In Proc. of WMT, Edinburgh, UK, July 2011.

Och, Franz Josef and Hermann Ney. A systematic comparison of various statistical alignment models. Computational Linguistics, 29(1):19-51, 2003.

Papineni, Kishore, Salim Roukos, Todd Ward, and Wei-Jing Zhu. BLEU: a method for automatic evaluation of machine translation. In Proc. of ACL, pages 311-318, Philadelphia, Pennsylvania, 2002. URL http://dx. doi . org/10.3115/1073083. 1073135.

\section{Address for correspondence:}

Ondřej Bojar

bojar@ufal.mff.cuni.cz

UK MFF ÚFAL

Malostranské náměstí 25

11800 Praha 1, Czech Republic 